\title{
Opportunities \& Challenges for Digital Health Solutions
}

'Darin Oppenheimer, Suraj Ramachandran, George Cusatis \& Daniel Visco, Merck, Sharpe \& Dohme Corp., a subsidiary of Merck \& Co, Inc.

Background

There is no question that the medical device and pharmaceutical industries are embarking on a technological journey focused on the utilization of digital health technology.

This journey is the convergence of several regulated industries coupled with information technology which has created novel approaches to the future of healthcare and medicine. We can see first hand that our dependency on technology provides the ideal conduit for further utilization of our smartphones, tablets, and personal computers.

This utilization of our daily technology has created a digital healthcare sector which is often thought of as products utilizing telemedicine, smart products, disease management, therapy reminders, and support message to advance diagnosis and treatment ${ }^{1}$.

\section{Introduction}

While this journey is casting light on new opportunities that were once perceived to be more science fiction than science, these rapid advancement can present new challenges for the interconnected industries - One of the challenges we will be discussing is specifically surrounding the effectiveness of risk management through regulatory expectations and cybersecurity.

- Risk management is not a new topic or methodology to these well established industries as each one has a different approach to managing and mitigating risk
Opportunities \& Challenges

- On August 29, 2017, the FDA issued a Safety health care providers about the release of a firmware update to address cybersecurity vulnerabilities update to address cybersecurity vulnerabilities implantable cardiac pacemakers. The firmware update continues Abbott's efforts to mitigate

independent research firm in 2016. On January 9, 2017, the FDA issued a Safety Communication confirming vulnerabilities in St. Jude Medical's implantable cardiac devices and Merlin@home Transmitter. The FDA became aware of cybersecurity vulnerabilities in these devices after an independent research firm - On July 31 2015, Hospira and an independent researcher confirmed that it is possible to access the Symbiq Infusion System remotely through a hospital's network

- On May 13, 2015, the FDA issued a Safety Communication on vulnerabilities of Hospira LifeCare PCA3 and PCA5 Infusion Pump Systems. The FDA and Hospira became aware of cybersecurity vulnerabilities in these infusion systems after an independent researcher released information about these vulnerabilities.

These examples highlight some of the challenges facing manufacturers today.

- The importance of implementing the above structure is apparent with the examples of the cyber incidents

- A cyber-attack on a regulated or non-regulated device could impact actual patient safety and patient care.

care providers about the ratients and additional firmware update to address premature battery depletion and confirmed cybersecurity vulnerabilities identified in Abbolt's (Iormenly St. Jude Medical) implantable cardiac devices

\section{Risk Management} released information about these vulnerabilities.

Since 2011, FDA has issued several guidance documents to provide clarity on how various digital technologies should be regulated. These polices addressed a range of products such as Mobile Applications, Medical Device Data Systems (MDDS), and Electronic Health Records (EHRs).

\section{Industry Best Practices}

The Health Care Industry Cybersecurity Task Force in the US published best practices for organizations to manage these new risks

- Define and streamline leadership,

governance, and expectations for health care industry cybersecurity.

- Increase the security and resilience of medical devices and health IT.

- Develop the health care workforce capacity necessary to prioritize and ensure cybersecurity awareness and technical capabilities.

- Increase health care industry readiness through improved cybersecurity awareness and education.

- Identify mechanisms to protect research and development efforts and intellectual property rom attacks or

- Improve information sharing of industry threats, weaknesses, and mitigations.

\section{Conclusion}

- It is unrealistic that a digital health product, or any product that incorporates technology for that matter, will completely be devoid of risks.

From a regulatory perspective, the proliferation of digital health technology has ignited numerous changes in legislation.

Correspondence to: Darin.Oppenheime@@Merck.com ${ }^{1}$ Global Regulatory Affairs and Clinical Safety, Device \& Digital
Health
- As products continue to evolve and connect patients, physicians, and manufacturers to health information (not to mention to each other) it is important to understand that there should always be an awareness of the potential risks that may exist and controls in place to address those risks.

- However regardless of a digital health product's regulatory status, ensuring that all identified risks, especially those relating to cybersecurity, are adequately addressed will go a long way to ensuring we continue to move forward in this new age with products that are safe, and do not compromise both users or the user's data.

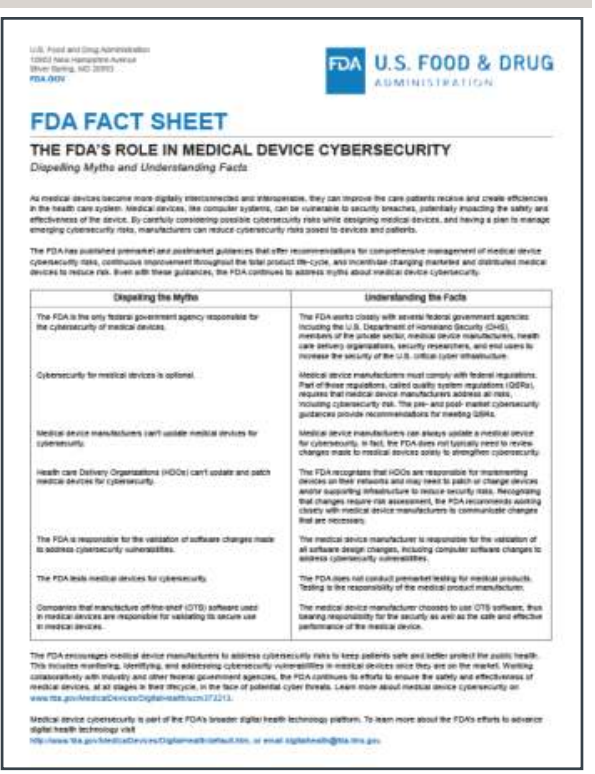

de Jongh T, Gurol-Urganci I, Vodopivec-Jamsek V, Car J,
Atun R. Mobile phone messaging for facilitating self-

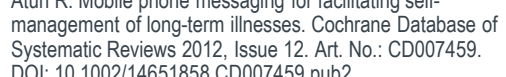
DOl: 10.1002/14651858.CD007459.pu 2. 